\title{
Idiopathic chronic fatigue in older adults is linked to impaired mitochondrial content and biogenesis signaling in skeletal muscle
}

\author{
Nicholas R. Wawrzyniak ${ }^{1}$, Anna-Maria Joseph ${ }^{2}$, David G. Levin ${ }^{1}$, David M. \\ Gundermann'2, Christiaan Leeuwenburgh ${ }^{2}$, Bhanuprasad Sandesara ${ }^{2}$, Todd $M$. \\ Manini ${ }^{2}$ and Peter J. Adhihetty ${ }^{1}$ \\ ${ }^{1}$ Department of Applied Physiology and Kinesiology, University of Florida, Gainesville, Florida, USA \\ 2 Department of Aging and Geriatric Research, Division of Biology of Aging, University of Florida, Gainesville, Florida, USA \\ Correspondence to: Peter J. Adhihetty, email: padhihetty@ufl.edu \\ Todd M. Manini, email: tmanini@ufl.edu
}

Keywords: fatigue; skeletal muscle; mitochondria; PGC-1a; Gerotarget

Received: January 11,2016 Accepted: June 09, 2016

Published: July 18, 2016

\section{ABSTRACT}

Fatigue is a symptom of many diseases, but it can also manifest as a unique medical condition, such as idiopathic chronic fatigue (ICF). While the prevalence of ICF increases with age, mitochondrial content and function decline with age, which may contribute to ICF. The purpose of this study was to determine whether skeletal muscle mitochondrial dysregulation and oxidative stress is linked to ICF in older adults. Sedentary, old adults ( $n=48$, age $72.4 \pm 5.3$ years) were categorized into ICF and non-fatigued (NF) groups based on the FACIT-Fatigue questionnaire. ICF individuals had a FACIT score one standard deviation below the mean for nonanemic adults $>65$ years and were excluded according to CDC diagnostic criteria for ICF. Vastus lateralis muscle biopsies were analyzed, showing reductions in mitochondrial content and suppression of mitochondrial regulatory proteins Sirt3, PGC-1a, NRF-1, and cytochrome $c$ in ICF compared to NF. Additionally, mitochondrial morphology proteins, antioxidant enzymes, and lipid peroxidation were unchanged in ICF individuals. Our data suggests older adults with ICF have reduced skeletal muscle mitochondrial content and biogenesis signaling that cannot be accounted for by increased oxidative damage.

\section{INTRODUCTION}

"As a self-reported measure, fatigue is complex and multidimensional and, not surprisingly, has been defined in a number of ways: as a feeling that interferes with usual functioning and has a multifactorial origin, as a sense of diminished energy and increased need to rest, and as physical or mental weariness resulting from exertion. As such, definitions of fatigue frequently vary across studies, across diseases, and even between investigators and patients," [1].

Systemic fatigue (here on referred to as "fatigue") typically manifests itself in association with a wide variety of clinical pathologies including cancer, congestive heart failure, sleep disorders, mental disorders, and fibromyalgia
[1]. Chronic fatigue presents as an overall lack of systemic energy that persists for numerous months and cannot be alleviated by prolonged rest, leading to functional declines in daily living activities [1-3]. Additionally, the clinical definition of chronic fatigue syndrome (CFS) must exhibit at least four of the following symptoms to qualify as CFS, which include: impairment of short-term memory or concentration, sore throat, tender lymph nodes, muscular pain, joint pain, headaches, unrefreshing sleep, and post-exertional malaise. However, there are individuals who report or exhibit sustained fatigue in the complete absence of any underlying medical conditions and this is referred to as "idiopathic chronic fatigue" (ICF), which is different from CFS. ICF is defined as clinically diagnosed psychological and physiological fatigue with 
no known underlying pathologies and does not need to meet four of the six criteria of CFS [2]. Although no known etiology exists for ICF, it has been speculated to stem from metabolic abnormalities within various tissues, particularly skeletal muscle, but the molecular details involved have yet to be elucidated $[1,4,5]$. These tissues decline as part of the "aging process," while the incidence of ICF is higher in elderly individuals [1], suggesting there may be age-related metabolic changes that could exacerbate symptoms of fatigue.

Abnormalities in mitochondrial regulation and function in muscle tissue are potential areas worth exploring for a causal relation to ICF since this organelle plays an integral role in regulating bioenergetics and oxidative capacity, particularly in high energy-demanding tissues [6]. Mitochondrial content is maintained via a process termed mitochondrial biogenesis and involves the coordination of the nuclear and mitochondrial genomes. This is orchestrated by the transcriptional coactivator peroxisome proliferator-activated receptor gamma coactivator-1 alpha (PGC-1 $\alpha$ ) that acts to increase the expression of numerous genes involved in mitochondrial biogenesis including nuclear respiratory factors 1 and 2 (NRF-1, NRF-2) and mitochondrial transcription factor A (TFAM) [7-11]. Additionally, mitochondrial morphology proteins of fission (Drp-1, Fis-1) and fusion (Mfn-1, Mfn2) are critical for maintaining cycles of mitochondrial turnover, and recycling of mitochondrial constituents [12-15]. Mitochondrial biogenesis is also associated with nascent formation of electron transport chain (ETC) complexes, which are crucial for the production of ATP (energy) via cellular respiration. Thus, dysregulation of ETC complexes may be linked to ICF because chronic fatigue is often described as a "lack of energy". Impairments in mitochondrial biogenesis, regulatory processes and overall mitochondrial function are involved in the pathophysiology of diabetes, Alzheimer's, Parkinson's, Huntington's disease, sarcopenia, and normal aging [16-22]. However, it is currently unknown whether impairments in mitochondrial biogenesis and/or mitochondrial quality control pathways contribute to the etiology of ICF in older adults.

Aging without overt disease is associated with significant impairments in the size, function, and metabolic profile of skeletal muscle [20, 23-25]. Although there are a variety of underlying mechanisms contributing to age-related skeletal muscle dysfunction, aberrations in mitochondrial function and regulation appears to be a central factor because of the mitochondrion's role in cellular energy production (i.e. ATP), origin of damaging free radicals, impaired autophagy and mitochondriallymediated apoptosis [19, 26-30]. Currently, only a few studies have investigated whether mitochondrial abnormalities contribute towards dysregulated muscle bioenergetics in younger patients with chronic fatigue. Smits et al. (2011) showed that skeletal muscle from younger patients with chronic fatigue syndrome (CFS) exhibited no change in mitochondrial respiratory chain complex activities or ATP production but overall mitochondrial content was significantly reduced compared to controls [31]. Additionally, a study investigating middle-aged patients diagnosed with CFS found reduced mitochondrial function, higher levels of lipid peroxidation, but similar numbers of mitochondria in white blood cells when compared to healthy controls [32]. Furthermore, patients with CFS have elevated markers of oxidative damage in muscle [33] and plasma [34], which may stem from the overproduction of damaging free radicals resulting from impaired mitochondrial function. Despite this limited evidence for mitochondrial involvement in CFS, there are no studies to date that have comprehensively investigated mitochondrial function and regulation in an aged population with ICF [35].

In the present study, we tested the hypothesis that older adults presenting with ICF would exhibit lower mitochondrial content, function, and impaired regulation in skeletal muscle when compared to NF counterparts. Specifically, we hypothesized that older adults presenting with ICF would exhibit lower skeletal muscle mitochondrial enzymatic activity, number, gene expression, bioenergetic regulation and quality control (fission \& fusion) compared to age-matched NF older adults. Importantly, our study used stringent eligibility criteria, analogous to the criteria for diagnosing CFS such that outright and subclinical comorbidities commonly associated with fatigue were excluded from the study to minimize the contamination of underlying medical conditions.

\section{RESULTS}

\section{Participant characteristics}

The main participant characteristics are provided in Table 1. A total of 48 individuals (age $72.4 \pm 5.3$ years) participated in the study that included 20 idiopathic chronically fatigued (ICF) individuals and 28 non-fatigued (NF) age-matched counterparts. Most participants were Caucasian (95\%) and consisted of a similar number of males $(n=26)$ and females $(n=22)$. As expected, fatigued participants had higher FACIT-F scores, but there were no differences in age, gender, and ethnicity were observed between the two groups. The ICF group had a slightly higher (12\%) body mass index, reported more depressionlike symptoms on the CES-D and was more likely to be treated for hypertension. Regarding blood counts, the ICF group had higher levels of neutrophils and lower aspartate transaminases, lymphocytes, basophils and red cell distribution. 
Table 1: Descriptive participant characteristics.

\begin{tabular}{|c|c|c|c|}
\hline $\begin{array}{l}\text { Characteristics } \\
\text { mean (SD) or No. (\%) }\end{array}$ & $\begin{array}{l}\text { NF } \\
(n=28)\end{array}$ & $\begin{array}{l}\text { ICF } \\
(n=20)\end{array}$ & $p$-value \\
\hline Age (yrs.) & $72.4(4.9)$ & $72.4(5.9)$ & 0.99 \\
\hline Caucasian & $26(92.9)$ & $20(100.0)$ & 0.48 \\
\hline Female & $13(48.2)$ & $9(45.0)$ & 0.83 \\
\hline College education & $22(78.6)$ & $17(85.0)$ & 0.57 \\
\hline Live alone & $10(35.7)$ & $5(25.0)$ & 0.43 \\
\hline MMSE score (0-30) & $28.4(1.7)$ & $28.2(1.9)$ & 0.71 \\
\hline Body Mass Index $\left(\mathrm{kg} / \mathrm{m}^{2}\right)$ & $25.5(4.1)$ & $27.7(2.6) *$ & 0.04 \\
\hline FACIT-F score (0-50) & $49.5(2.5)$ & $24.3(6.1) *$ & $<0.01$ \\
\hline Excellent/very good health & $26(92.9)$ & $13(65.0) *$ & 0.02 \\
\hline Unable to walk one mile & $1(3.6)$ & $2(10.0)$ & 0.36 \\
\hline CES-D score (0-60) & $3.68(3.9)$ & $10.1(4.4) *$ & $<0.01$ \\
\hline History of cardiovascular disease & $4(14.3)$ & $3(15.0)$ & 0.95 \\
\hline Osteoarthritis & $2(7.14)$ & $4(20.00)$ & 0.18 \\
\hline History of cancer & $7(25.0)$ & $7(35.0)$ & 0.45 \\
\hline Hypertension & $11(40.7)$ & $14(70.0) *$ & 0.05 \\
\hline Diabetes & $3(10.7)$ & $5(25.0)$ & 0.19 \\
\hline Fasting glucose (mg/dL) & $95.6(12.8)$ & $106.6(21.7) *$ & 0.04 \\
\hline Albumin (g/dL) & $4.4(0.2)$ & $4.5(0.3)$ & 0.07 \\
\hline Aspartate transaminase (IU/L) & $25.8(6.6)$ & $22.1(3.0)$ & 0.02 \\
\hline Alanine transaminase (IU/L) & $24.4(9.3)$ & $21.2(5.8)$ & 0.18 \\
\hline Platelets & $252.4(52.2)$ & $243.6(60.6)$ & 0.59 \\
\hline White blood cell count & $5.8(1.6)$ & $6.0(0.8)$ & 0.64 \\
\hline Neutrophils & $56.3(7.7)$ & $61.8(5.6)$ & 0.01 \\
\hline Lymphocytes & $30.0(7.4)$ & $26.1(4.8) *$ & 0.04 \\
\hline Monocytes & $6.6(1.6)$ & $6.5(1.5)$ & 0.83 \\
\hline Eosinophils (\%) & $3.7(2.0)$ & $3.0(2.0)$ & 0.27 \\
\hline Basophils (\%) & $0.9(0.5)$ & $0.6(0.3)$ & 0.02 \\
\hline
\end{tabular}




\begin{tabular}{|l|l|l|l|}
\hline Hemoglobin $(\mathbf{g} / \mathbf{d L})$ & $14.3(0.9)$ & $14.2(1.3)$ & 0.80 \\
\hline Hematocrit & $42.8(2.7)$ & $42.7(3.7)$ & 0.96 \\
\hline Red blood cells & $4.6(0.4)$ & $4.6(0.3)$ & 0.75 \\
\hline Corpuscular volume & $93.5(5.8)$ & $92.3(4.6)$ & 0.45 \\
\hline Corpuscular hemoglobin & $31.2(1.8)$ & $30.6(1.6)$ & 0.28 \\
\hline Red cell distribution & $12.5(0.7)$ & $13.0(0.7) *$ & 0.04 \\
\hline
\end{tabular}

MMSE $=$ Mini-Mental State Exam. FACIT-F $=$ Functional Assessment of Chronic Illness Therapy Fatigue scale. CES-D = Center for Epidemiologic Studies Depression scale. $\bullet$ CVD disease defined as a history of heart attack, coronary/myocardial infarction, stroke/brain hemorrhage, heart failure/congestive heart failure or abnormal heart rhythm. Values are reported as means \pm SD for continuous variables and or $\mathrm{n}(\%)$ for categorical variables. Significance set at ${ }^{*} P<0.05$.

\section{Skeletal muscle mitochondrial content}

Reductions in mitochondrial content and bioenergetics are a common feature of skeletal muscle dysfunction in a number of conditions including but not limited to aging, cachexia, and sarcopenia. Cytochrome $c$ oxidase (COX) activity, a common marker of mitochondrial content [36] was significantly lower $(18.4 \%)$ in ICF individuals compared to NF (Figure 1).

\section{Mitochondrial biogenesis signaling}

AMPK (5' adenosine monophosphate-activated protein kinase) activation, measured by phosphorylatedAMPK over total-AMPK, was not altered in ICF individuals compared to the NF group (Figure 2). Levels of the mitochondrially-localized Sirt3 protein were lower $(18.1 \% ; P<0.05)$ in ICF individuals compared to NF, while there was a trend for lower levels of Sirt1 protein $(P=0.08$; Figure 2$)$. PGC- $1 \alpha$ expression was also

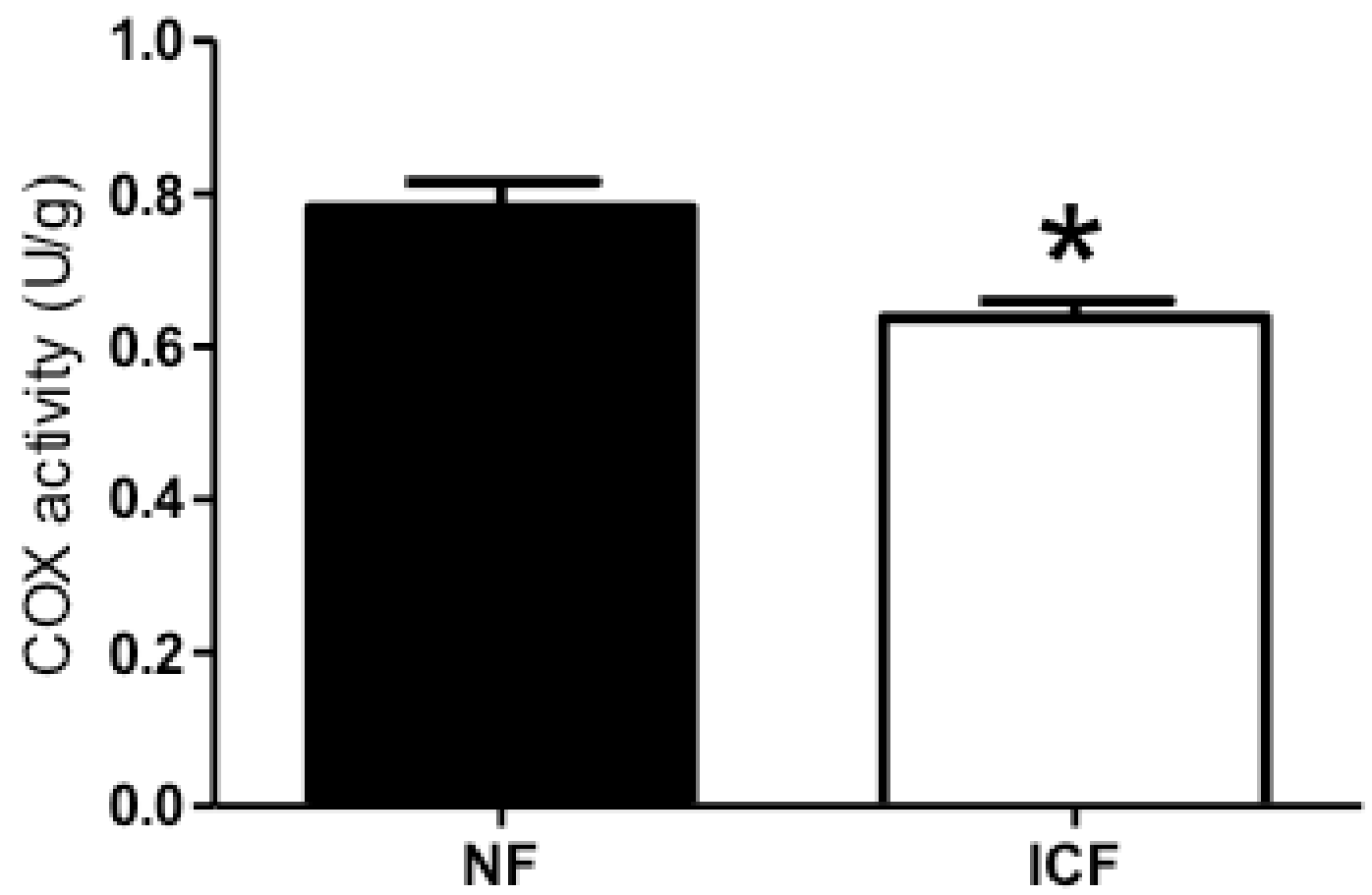

Figure 1: Mitochondrial content. Mitochondrial content was measured by Cytochrome $c$ oxidase (COX) activity $(n=17-26$ per group) from vastus lateralis muscle biopsies of ICF and NF individuals. Values are means \pm SEM with significance set at $* P<0.05$. 
significantly lower $(37.4 \% ; P<0.05)$ in ICF individuals (Figure 2), as were levels of its downstream target, NRF-1 (19.4\%; $P<0.05$; Figure 2). No differences were observed in TFAM, but there was a trend for lower expression of cytochrome $\mathrm{c}$ in the ICF group $(P=0.09$; Figure 2$)$.

\section{Electron transport chain complexes}

Levels of ETC complexes I-III were not altered in ICF individuals compared to NF counterparts. However, Complex IV (cytochrome c oxidase) and Complex V (ATP
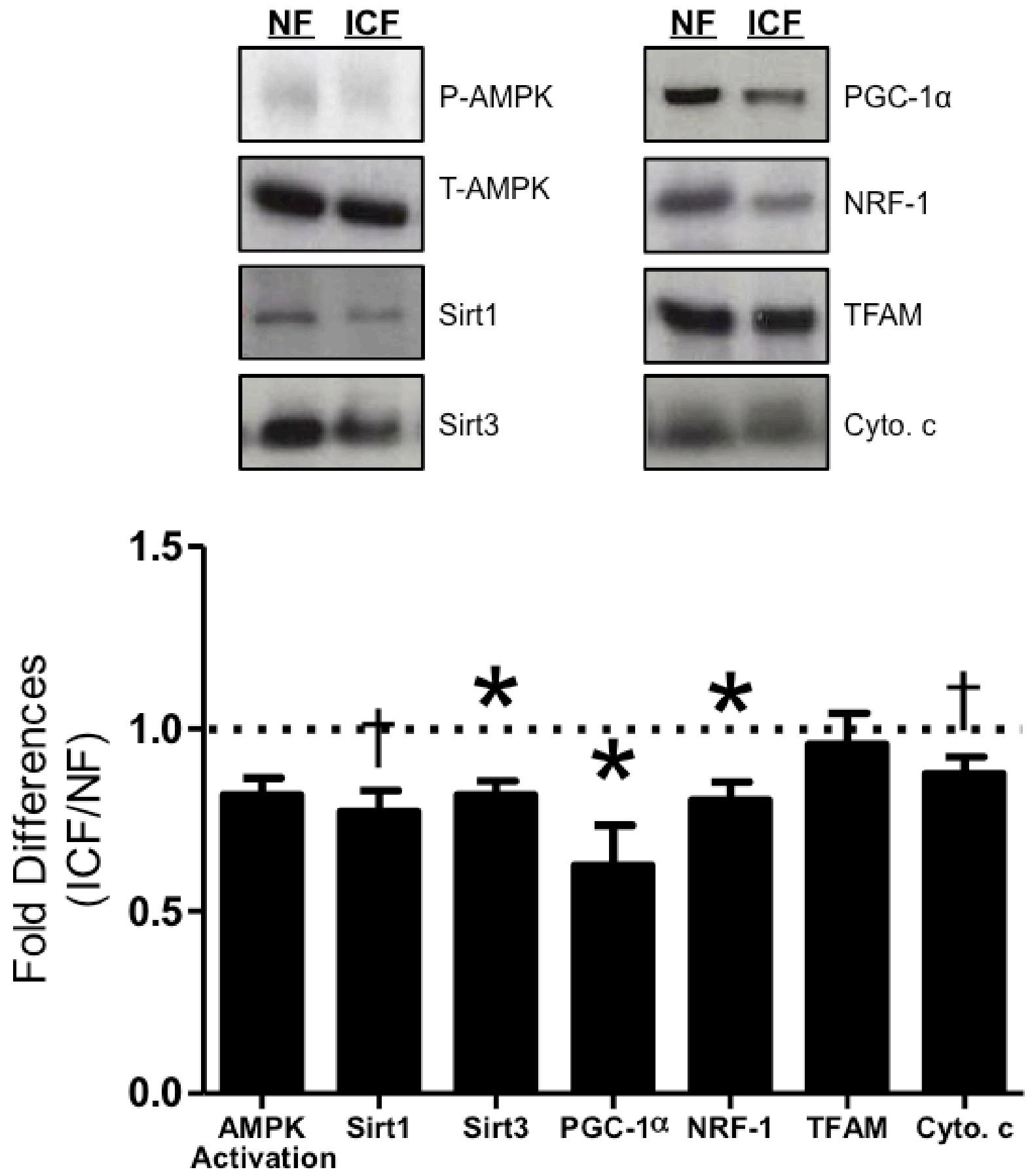

Figure 2: Mitochondrial biogenesis signaling. Western blot analysis of key biogenesis signaling proteins including AMPK $=5$ ' adenosine monophosphate-activated protein kinase; Sirt1 = sirtuin 1; Sirt3 = sirtuin 3; PGC-1 $\alpha=$ peroxisome proliferator-activated receptor gamma coactivator-1 alpha; NRF-1 = nuclear respiratory factor 1 ; TFAM = mitochondrial transcription factor A; Cyto. $\mathrm{c}=$ cytochrome $\mathrm{c}$. AMPK activation was determined as ratio of phosphorylated protein (p-AMPK) compared to total protein (t-AMPK). Graphical summary of repeated experiments is shown below. Values are represented as means $\pm \mathrm{SEM} ; n=16-28$ per group. ${ }^{*} P<0.05 ; \uparrow P<0.10$. Data are expressed as fold difference (ICF/NF). 
synthase) were both significantly lower $(22.3 \%, P<0.05$; $9.9 \% P<0.05$ ) in ICF patients (Figure 3 ).

\section{Mitochondrial morphology proteins}

ICF and NF individuals had similar levels of mitochondrial fusion proteins mitofusin 1 (Mfn-1) and mitofusin 2 (Mfn-2), and mitochondrial fission proteins dynamin-related protein 1 (Drp-1) and fission protein 1 (Fis-1) (Figure 4).

\section{Oxidative damage and antioxidant capacity}

Lipid peroxidation, measured as 4-hydroxynonenol (4-HNE), was similar between the ICF and NF groups

(Figure 5). Although manganese superoxide dismutase
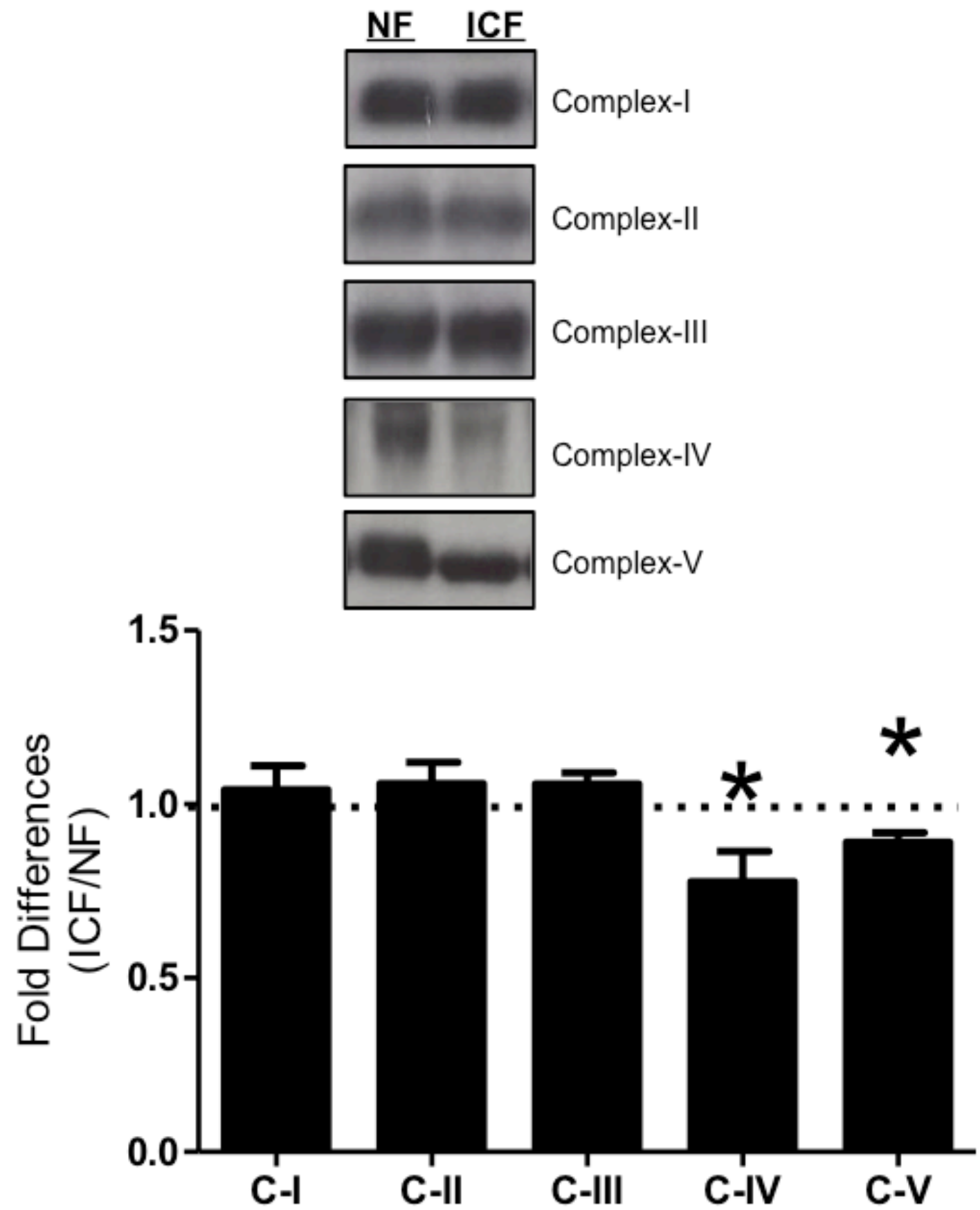

Figure 3: ETC complexes. Expression levels from subunit proteins of ETC complexes I-V were measured via western blot. These included CI - subunit NDUFB8 (nuclear encoded), CII - 30kDa (nuclear), CIII - core protein 2 (nuclear); CIV - subunit I (mtDNA encoded); $\mathrm{CV}$ - alpha subunit (nuclear). Summary of repeated experiments is shown below. Values are represented as means $\pm \mathrm{SEM} ; n=18-28$ per group. Significance set at $* P<0.05$. Data are expressed as fold difference (ICF/NF). 
(MnSOD) tended to be lower in ICF individuals $(9.9 \%, P$ $=0.07)$, there were no statistically significant differences to support antioxidant species catalase (Cat) and glutathione peroxidase (GPx) as a major factor explaining fatigue in this sample population (Figure 5).

\section{DISCUSSION}

There is limited mechanistic data to explain the presentation of ICF in older adults [4, 5]. However, mitochondrial dysfunction represents a plausible underlying mechanism for ICF since impaired function of these energy producing organelles in highly metabolic

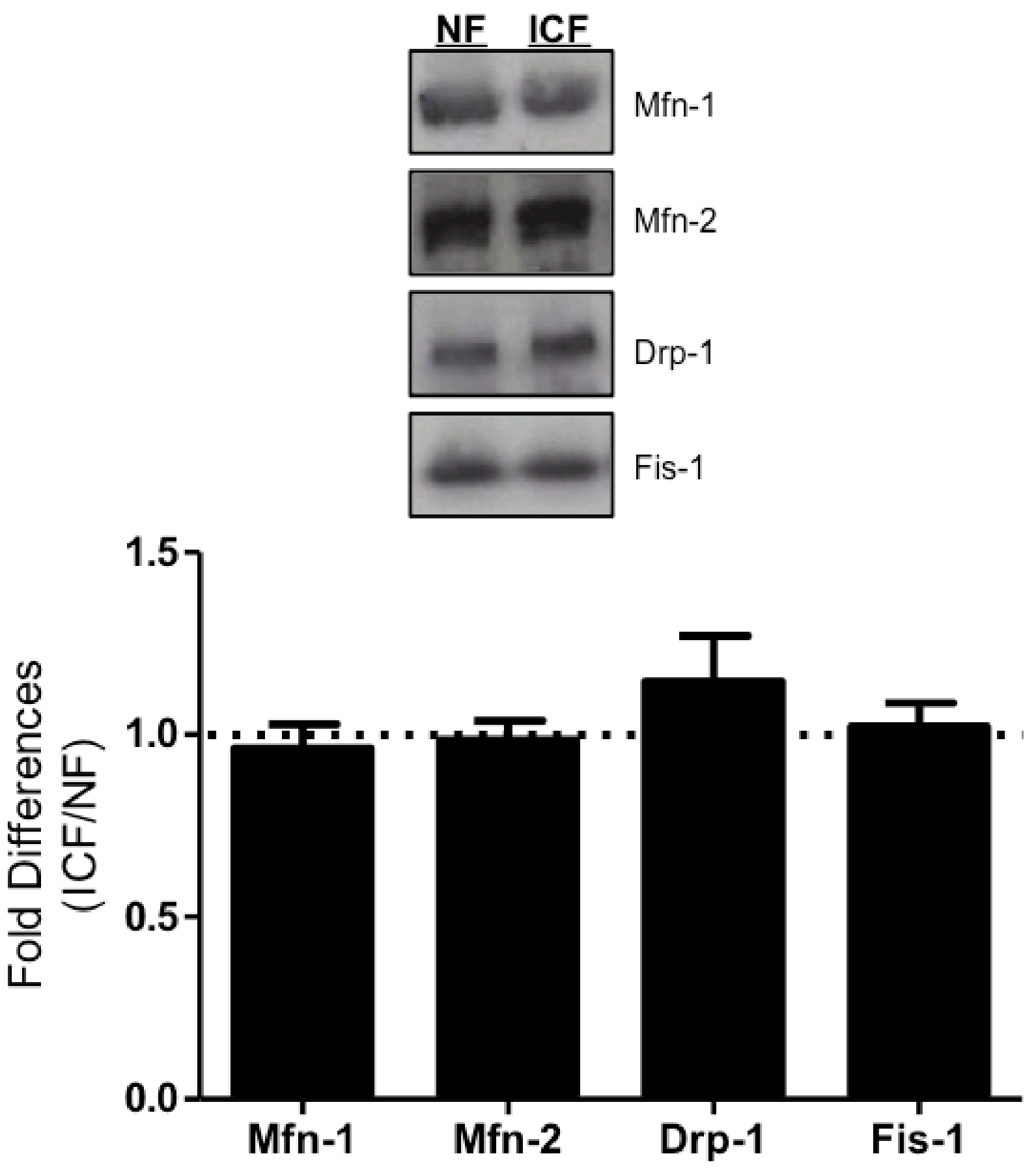

Figure 4: Mitochondrial morphology. Mitochondrial fusion (Mfn-1 = mitofusin 1; Mfn-2 = mitofusin 2) and fission proteins (Drp-1 = dynamin-related protein 1; Fis-1 = fission protein 1) were measured via western blot. Graphical summary of repeated experiments is shown below. Values are represented as means \pm SEM; $n=15-27$ per group. Significance set at $* P<0.05$. Data are expressed as fold difference $(\mathrm{ICF} / \mathrm{NF})$. 
tissues, such as muscle, is associated with numerous disease states and conditions, including aging. We hypothesized that older adults presenting with ICF would exhibit lower skeletal muscle mitochondrial enzymatic activity, number, gene expression, bioenergetic regulation and quality control (fission \& fusion) compared to agematched NF older adults. To our knowledge, this is the first study to provide a detailed mechanistic analysis of mitochondrial content and signaling in skeletal muscle of older adults with ICF. The results suggest that older adults
NF ICF

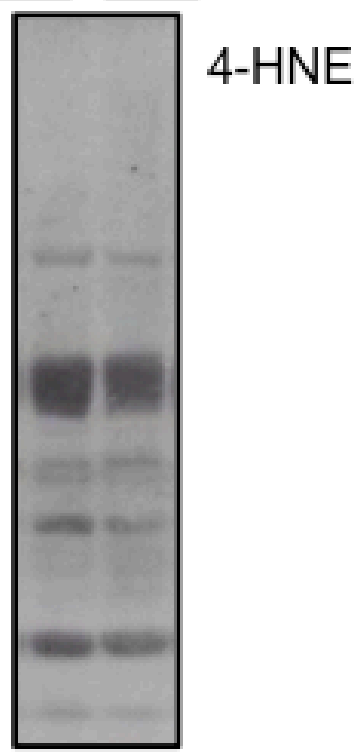

NF ICF

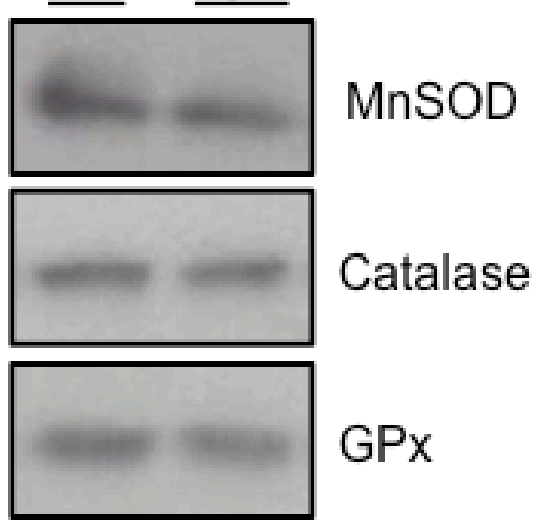

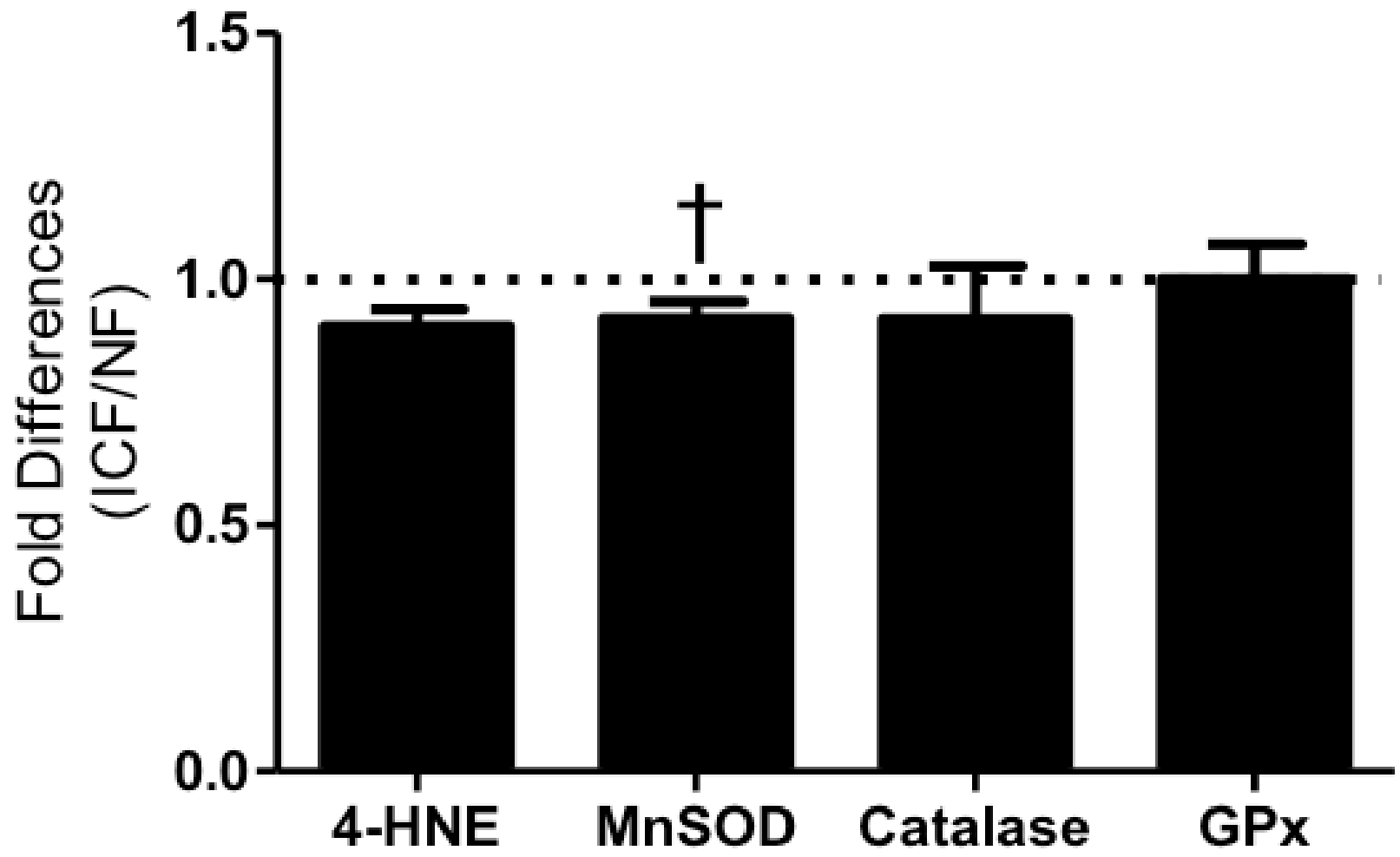

Figure 5: Oxidative damage and antioxidants. Lipid peroxidation (4-HNE = 4-hydroxynonenal) and expression of critical antioxidant enzymes $(\mathrm{MnSOD}=$ Manganese superoxide dismutase; Cat = catalase; and GPx = glutathione peroxidase) were measured via western blot. Summary of repeated experiments are depicted below. Values are represented as means \pm SEM; $n=18-27$ per group. $\dagger P<$ 0.10. Data are expressed as fold difference (ICF/NF). 
with ICF exhibit greater mitochondrial dysregulation that may reduce the muscle's ability to produce ATP and that this may contribute towards the etiology and symptoms associated with ICF.

Numerous mitochondrially-mediated mechanisms are involved with age-associated reductions in energy homeostasis, redox status, mitophagy, mitochondrial dynamics and apoptosis [17, 18, 20, 37-42]. In this study, we found older adults with ICF had reduced overall mitochondrial content in muscle compared to their agematched NF counterparts. The explanations for these findings could be diverse, and one of which may be related to exercise and overall daily physical activity that is clearly associated with overall mitochondrial content and mitochondrial-mediated adaptations [6, 43-47]. However, both groups of participants were considered sedentary after being screened for engaging in less than 20 minutes of structured physical activity per week. Additionally, significant results were unchanged after adjusting for the variability in light, moderate and vigorous physical activity in a subset of 28 participants who wore hip accelerometers (data not shown). Despite equally sedentary behaviors, ICF individuals presented with slightly higher (but statistically significant) BMI levels than their NF counterparts. Individuals with Class II Obesity (BMI > $35)$ were previously shown to have reductions in skeletal muscle mitochondrial content, specifically cytochrome c oxidase levels $(-32 \%)$, compared to lean $(\mathrm{BMI}<25)$ agematched counterparts [48]. Thus, slight elevations in BMI of ICF individuals may contribute to a cyclical pattern of increasingly sedentary behaviors, reduced mitochondrial content, and increased sensations of fatigue. Examination of correlations with each mitochondrial marker to BMI revealed that only TFAM was significantly correlated to BMI $(r=0.34, p=0.02)$, while all others were lower than $r=0.15(p>0.30)$. Multiple regression models showed that differences between groups in mitochondrial markers remained unchanged when adjusting for BMI (data not shown). Lastly, we did observe slightly different immune profiles (basophils, lymphocytes, and neutrophils) between the two groups. However, it should be noted that these levels are well within normal ranges and would not typically be a medical concern. For example, the normal range for neutrophils is $35-80 \%$. The ICF group had a mean value of $61.8 \%$, which is well within normal ranges. This was also true for lymphocytes and basophils. Therefore, we don't believe these differences are a cause of fatigue symptoms, but may be related to anxiety caused by fatigue symptoms, which is commonly noted in older adults with depression $[49,50]$. In all, our data indicate the magnitude of mitochondrial dysregulation is sufficient to detect differences between fatigue states, which are noteworthy considering that fatigue, when measured as a general state via a questionnaire, is complex and multidimensional.

Mitochondrial content in skeletal muscle is largely dictated by mitochondrial biogenesis processes controlled primarily via PGC-1 $\alpha[6,9,10,51,52]$. Aged muscle is associated with reductions in $\mathrm{PGC}-1 \alpha$ expression levels [53], mitochondrial content, and mitochondrial function [17, 37, 46, 54]. Transgenic overexpression of PGC-1 $\alpha$ in skeletal muscle yields reduced dysfunction and suppressed cachexia with age, leading to improved strength and endurance capacity $[45,55]$. In our study, we found significant reductions in PGC- $1 \alpha$ protein levels and in its upstream activator (Sirt1) and downstream effectors (NRF-1, Sirt3) in older adults with ICF. Because Sirt3 is dependent upon the expression of PGC-1 $\alpha$ to orchestrate antioxidant expression and mediate cellular respiratory status, such reductions can ultimately lead to disruption of cellular energy homeostasis and oxidative capacity [56]. Furthermore, activation of skeletal muscle PGC$1 \alpha$ was recently shown to reduce depressive symptoms, of which there is overlap with ICF symptoms, in rodents by blocking the kynurenine pathway of tryptophan degradation. PGC- $1 \alpha$ exerts these effects by activating kynurenine acetyltransferases to convert kynurenine (a stress-induced metabolite able to cross the blood-brain barrier and contribute towards depression) into kynurenic acid (which is unable to cross the blood-brain barrier) and thus minimizing the manifestations of depressive symptoms in the brain [57]. In the present study, participants in the ICF group reported more depressive symptoms and thus had higher CES-D scores, but there were significant overlaps in the wording of the questions on the FACIT-F and CES-D questionnaires. This overlap made it difficult to delineate the differences between depression-like and fatigue-like symptoms. However, in accordance with our exclusion criteria, none of our participants were clinically diagnosed with depression. Interestingly, Kato, et al., reported, "elevated premorbid stress is a significant risk factor for chronic fatigue-like illness [in the general population]," [58]. Taken together, these data suggest that older adults with ICF have lower skeletal muscle PGC-1 $\alpha$, which may relate to increased fatigue symptoms through recently discovered pathways that connect muscle biomarkers to perceived fatigue symptoms in the brain.

Components of the ETC are integral to the mitochondrion's ability to maintain energy homeostasis. A vast majority of ETC proteins encoded by the mitochondrial genome are regulated by the important mitochondrial transcription factor, TFAM, which is controlled by PGC- $1 \alpha$ via activation of NRF-1. Despite significant decrements in PGC- $1 \alpha$ and NRF-1, TFAM expression levels were not altered and this coincided with unchanging expression of proteins representing complexes I-III in ICF compared to NF. However, marked decreases in the expression of complexes IV and V of the ETC were exhibited with ICF. Interestingly, these two complexes have a greater proportion of their proteins encoded by the nuclear genome compared to complexes I-III and 
reductions in these two complexes have significant impact on mitochondrial oxidative capacity and energy production [54]. These reductions in mitochondrial signaling proteins were relatively homogenously correlated to the 13 individual FACIT-F questions (data not shown), indicating that the summed FACIT-F score was representative of the questions individually. Collectively, our data indicate significant decreases in upstream mitochondrial signaling (i.e. PGC-1 $\alpha$ ) leads to deleterious alterations of downstream ETC complexes, which may impair the ATP producing capacity of the mitochondria because these complexes play a central role in chemiosmotic coupling [19, 59-61].

Mitochondria are a significant source of cellular reactive oxygen species (ROS) production which beyond a certain cellular threshold can lead to oxidative stress for the cell and cause damage to intracellular macromolecules such as proteins, lipids and nucleic acids. Oxidative stress and other cellular perturbations can evoke processes of fission and fusion to alter mitochondrial ultrastructure in order to preserve the viability of the organelle [12, 15, 19, 22, 62-64]. Mitochondrial morphology proteins were not different between the ICF and NF groups despite reductions in mitochondrial biogenesis signaling molecules, a finding that is corroborated by previous data from studies in PGC- $1 \alpha$ knockout animals [36]. This suggests that mitochondrial morphology is not a process that is altered with ICF, but electron microscopy of mitochondria would be required to confirm this finding. To investigate whether oxidative damage and/or oxidative stress may contribute to ICF, we analyzed lipid peroxidation measured as 4-hydroxynonenol (4-HNE) and the expression of the antioxidant enzymes manganese superoxide dismutase (MnSOD), catalase, and glutathione peroxidase. Our data only showed a modest decrement in MnSOD with all other markers indicating no significant differences in oxidative damage or antioxidant capacity with ICF. Taken together, these findings suggest that neither oxidative damage nor alterations in mitochondrial morphology and ultrastructure appear to contribute to mitochondrial abnormalities with ICF. Thus, despite lower mitochondrial number, biogenesis signaling, and ETC enzymatic content with ICF, these impairments did not transfer to elevated oxidative damage and/or oxidative stress in muscle.

We have shown that reductions in overall mitochondrial content associated with impaired mitochondrial regulatory pathways appear to contribute towards ICF in older adults. Specifically, significant declines in the expression of upstream and downstream proteins associated with PGC-1 $\alpha$, as well as reduced PGC-1 $\alpha$ itself, may be an important factor in the lack of systemic energy in these subjects. This study was limited by our analyses and interpretations on a relatively small amount of muscle biopsy tissue available to perform more comprehensive biochemical assays.
Further studies investigating more detailed cellular and molecular mechanisms associated with ICF in older adults are warranted. Another limitation of this and previous epidemiological studies on ICF and aging is the possibility that self-reported fatigue in these studies are confounded by other subclinical conditions $[35,65,66]$, which we attempted to rectify by employing stringent exclusion criteria and blood work in line with the CDC case definition [2]. However, subclinical diseases not captured during the screening process may continue to contribute to ICF symptoms. Although poor nutrition could be a potential cause of fatigue, we did not measure dietary intake. However, it is likely to have a low effect because there was a relatively small difference in BMI between the two groups where the averages of both groups fell in the overweight range and BMI had little association with the mitochondrial markers. Lastly, while physical activity is clearly associated with marked adaptations in mitochondrial number and function, this study sought to remove this element by including sedentary individuals and excluding individuals who were physically active. However, future studies are needed to evaluate the impact that physical activity may have on the mitochondrial capacity in older adults with ICF. Additional studies are also warranted to investigate whether the aforementioned mitochondrial mechanisms are a cause or consequence of ICF, whether these abnormalities are localized to skeletal muscle or systemic, and if there is any crosstalk between the musculoskeletal and nervous systems which would amplify the sensations of ICF.

\section{CONCLUSIONS}

The aim of the present study was to investigate whether abnormalities in skeletal muscle mitochondria were associated with idiopathic chronic fatigue in older adults. More specifically, we hypothesized older adults with ICF exhibited 1) impairments in mechanisms regulating mitochondrial biogenesis signaling, 2) reductions in ETC complex expression, and/or 3) disruptions in the balance of oxidative damage and antioxidant capacity. Overall, our data points to impairments in mitochondrial number, biogenesis and electron transport chain activity as the primary perpetrators underlying the cellular mechanisms of ICF in older adults. Thus, interventions designed to target these mitochondrial impairments may be efficacious in combating fatigue and/ or the perceived sensations of fatigue. 


\section{EXPERIMENTAL METHODS}

\section{Participants}

A total of 61 sedentary men and women $65+$ years old were enrolled in the study. The following exclusionary criteria were created to limit disease conditions that are overtly related to fatigue symptoms: depressive symptoms $(C E S-D \geq 16)$, untreated sleep apnea, alcohol or substance abuse, body mass index $\geq 35 \mathrm{~kg} / \mathrm{m}^{2}$, degenerative neurological diseases, inflammatory disease, any pulmonary disease, cancer in past year, stroke in the past 6 months, fracture or joint replacement in past 6 months, insomnia being treated with hypnotic medication more than 2 nights/week, terminal illness, severe cardiac disease (NYHA Class III or IV congestive heart failure, clinically significant aortic stenosis, history of cardiac arrest, use of a cardiac defibrillator, or uncontrolled angina), severe sensory impairment, use of long-acting benzodiazepines or antipsychotic medication, living in a nursing home, actively participating in a formal exercise program within the past 3 months - defined as $20+$ minutes of formal exercise at least once a week, hypothyroidism: $\mathrm{T} 4<5 \mu \mathrm{g} /$ $\mathrm{dl}$ or normal T4 and elevated TSH $>20 \mu \mathrm{U} / \mathrm{ml}$, significant cognitive impairment defined as a known diagnosis of dementia or a Mini-Mental State Exam (MMSE) $<24$, uncontrolled hypertension, anemia, acute infection or fever within 4 weeks, abnormal blood potassium, sodium or calcium levels, and hyperglycemia. Prior to enrollment in the study, all participants provided written informed consent using documents approved by the University of Florida Institutional Review Board.

\section{Functional assessment of chronic illness therapy (FACIT) fatigue scale}

The FACIT Fatigue Scale (FACIT-F), a component of the FACIT Measurement System (http://www.facit. org/FACITOrg/Questionnaires) originally developed for patient reported outcomes in cancer and redeveloped for all chronic illnesses, was used to assess fatigue [67]. The scale assesses 13 self-reported items that relate to fatigue during daily activities over the past 7 days. It has been validated in a variety of disease conditions and in older adults [67-70]. Each item asked about a component of fatigue on a four point Likert scale $(4=$ not at all fatigued to $0=$ very much fatigued) [71]. The total FACIT Fatigue Score was calculated according to a standard protocol and referenced to normative data $[69,72]$ (http://www.facit. org/FACITOrg/Questionnaires). ICF and NF groups were established as values that were 2 standard deviations of normative data [69] with FACIT fatigue scores that fell within ranges of $\leq 35$ (ICF) and $\geq 42$ (NF). To ensure that fatigue symptoms were not transient, the FACIT-F was administered on two separate occasions separated by greater than one week. The correlation between the two FACIT-F questionnaires that were administered more than one week apart from each other was $0.94(p$ $<0.001)$ and a slope that followed the line of identity $($ Beta $=0.95)$. Participants who reported fatigue (or nonfatigue) symptoms outside the range were excluded for having transient fatigue that would not be consistent with idiopathic chronic fatigue.

\section{Muscle biopsies}

Skeletal muscle samples were obtained under local anesthesia from the vastus lateralis using the Bergstrom muscle biopsy technique [73]. Samples were immediately weighed and remaining tissue frozen in liquid nitrogen and stored at $-80 \mathrm{C}$ until further analysis.

\section{Cytochrome c oxidase (COX) enzyme activity}

Whole tissue was diluted to a 2-fold concentration in a buffer (0.1 M KH2PO4 + 2 mM EDTA, pH 7.2) and sonicated $(3 \times 5 \mathrm{~s})$ on ice. Following a brief spin, the supernatant was removed and enzyme activity determined by the maximal oxidation rate of completely reduced cytochrome c, evaluated as a change in absorbance at 550 $\mathrm{nm}$ using a multi-detection microplate reader (Synergy HT, Biotek Instruments, Winooski, VT) [36, 37].

\section{Immunoblotting}

Whole muscle tissue homogenates were prepared as previously described [36, 37]. Protein extracts from muscle homogenates were separated on $12-15 \%$ SDSpolyacrylamide gels and transferred to nitrocellulose membranes using an electroblotting transfer apparatus. Nitrocellulose membranes were blocked for $1 \mathrm{~h}$ with $5 \%$ skim milk in $1 \times$ TBS-T (Tris-buffered saline Tween-20: $25 \mathrm{mM}$ Tris- $\mathrm{HCl}, 1 \mathrm{mM} \mathrm{NaCl}, 0.1 \%$ Tween 20, pH 7.5). Membranes were incubated with primary antibodies overnight at $4{ }^{\circ} \mathrm{C}$ directed against phosphorylatedAMP-activated protein kinase- $\alpha$ (Thr172) (AMPK $\alpha$; 1:500; Cell Signaling; \#2531S), total-AMPK $\alpha$ (1:500; Cell Signaling, \#2532), Sirt1 (1:500; Cell Signaling, \#8469S), Sirt3 (1:100; Santa Cruz; sc-365175), PGC$1 \alpha$ (1:500; Calbiochem; \#516557), NRF-1 (1:500; Rockland Immunochemicals; \#200-4001-869), TFAM (1:500; Calbiochem; DR1071), cytochrome c (1:1500; Santa Cruz, sc-8385), Mfn-1 (1:1000; Sigma; M6319), Mfn-2 (1:1000; Sigma; M6444), Drp-1 (1:500; BioRad; 611112), Fis-1 (1:1000; Alexis Biochemicals; 210907-R100) 4-HNE (1:500; Abcam; ab46545), MnSOD (1:2000; Santa Cruz; sc-30080), Catalase (1:2000; Abcam; ab16731), GPx (1:1000; Abcam; ab22604), and electron 
transport chain subunit proteins using a total OXPHOS antibody cocktail (1:1000; Mitosciences; \#MS604). All commercially available antibodies have been extensively used in the literature. Ponceau S staining was used to normalize for the amount of protein loaded as previously described [24, 37]. Membranes were washed $(3 \times 5 \mathrm{~min})$ with TBS-T then blocked with the appropriate secondary antibody at room temperature for $1 \mathrm{~h}$, then washed again $(3 \times 5 \mathrm{~min})$ with TBS-T and signals were detected using enhanced chemiluminescence (ECL; Santa Cruz). Films were imaged and analyzed using Silk Scientific "Un-ScanItgel" gel analysis software measuring pixel density while correcting for background intensity.

\section{Other measurements}

Medical history and demographics (i.e. race, education, social status, mobility impairment, and selfrated health) were reported through clinic interview. Depressive symptoms were measured with the short form Center for Epidemiological Studies Depression Scale (CES-D) [74], and cognitive function was measured with the Mini Mental State Examination (MMSE) [75], Blood samples were used to ascertain sub-clinical conditions that may be responsible for fatigue symptoms (e.g. anemia). Blood was collected according to standard clinical protocols via venipuncture and assays were conducted in a Clinical Laboratory Improvement Amendments certified laboratory (CLIA certified).

\section{Statistical analysis}

Differences between groups of ICF and NF subjects were analyzed by using one-way analysis of variance (ANOVA). Correlations between variables were investigated using Pearson product moment correlation analyses. Differences were considered statistically significant if $P<0.05$ and $P$-values $<0.10$ were also noted. Data are presented as means \pm standard errors $(\mathrm{SE})$ unless otherwise noted.

\section{ACKNOWLEDGMENTS}

We would like to thank Linda M. Nguyen for technical assistance, Chaitalee Goswami for assistance with sample collection, and Ray A. Spradlin for assisting with tissue preparation.

\section{CONFLICTS OF INTEREST}

The authors declare no conflicts of interest.

\section{FUNDING}

This work was supported in part by the National Institute on Aging (award numbers P30AG028740 and R01 AG042525) and the National Heart, Lung, Blood Institute (award number: R01 HL121023). This work was supported by the National Institutes of Aging (award number R01 AG042525) and the University of Florida's Claude D. Pepper Center (award number P30 AG028740).

\section{Author Contributions}

C.L., T.M.M. and P.J.A designed the study; N.R.W and D.G.L. performed biochemical experiments; N.R.W., A-M.J., and P.J.A. analyzed and interpreted the data; B.S. collected patient samples; D.M.G. collected clinical data; N.R.W. drafted the manuscript; A-M.J., T.M.M., and P.J.A. helped develop the manuscript, and all authors reviewed the manuscript and gave their final approval.

\section{REFERENCES}

1. Alexander NB, Taffet GE, Horne FM, Eldadah BA, Ferrucci L, Nayfield S, Studenski S. Bedside-to-Bench Conference: Research Agenda for Idiopathic Fatigue and Aging: IDIOPATHIC FATIGUE AND AGING. J Am Geriatr Soc 2010; 58:967-975.

2. Fukuda K, Straus SE, Hickie I, Sharpe MC, Dobbins JG, Komaroff A. The chronic fatigue syndrome: a comprehensive approach to its definition and study. Ann Intern Med 1994; 121:953-959.

3. Holmes GP. Chronic Fatigue Syndrome: A Working Case Definition. Ann Intern Med 1988; 108:387.

4. Eldadah BA. Fatigue and Fatigability in Older Adults. PM\&R 2010; 2:406-413.

5. Vestergaard S, Nayfield SG, Patel KV, Eldadah B, Cesari M, Ferrucci L, Ceresini G, Guralnik JM. Fatigue in a Representative Population of Older Persons and Its Association With Functional Impairment, Functional Limitation, and Disability. J Gerontol A Biol Sci Med Sci 2009; 64A:76-82.

6. Hood DA. Invited Review: contractile activity-induced mitochondrial biogenesis in skeletal muscle. J Appl Physiol 2001; 90:1137-1157.

7. Handschin C, Spiegelman BM. Peroxisome ProliferatorActivated Receptor $\gamma$ Coactivator 1 Coactivators, Energy Homeostasis, and Metabolism. Endocr Rev 2006; 27:728735.

8. Lin J, Handschin C, Spiegelman BM. Metabolic control through the PGC-1 family of transcription coactivators. Cell Metab 2005; 1:361-370.

9. Wu Z, Puigserver P, Andersson U, Zhang C, Adelmant G, Mootha V, Troy A, Cinti S, Lowell B, Scarpulla RC, Spiegelman BM. Mechanisms Controlling Mitochondrial 
Biogenesis and Respiration through the Thermogenic Coactivator PGC-1. Cell 1999; 98:115-124.

10. Scarpulla RC. Metabolic control of mitochondrial biogenesis through the PGC-1 family regulatory network. Biochim Biophys Acta BBA - Mol Cell Res 2011; 1813:1269-1278.

11. Scarpulla RC. Transcriptional Paradigms in Mammalian Mitochondrial Biogenesis and Function. Physiol Rev 2008; 88:611-638.

12. Benard G, Bellance N, James D, Parrone P, Fernandez H, Letellier T, Rossignol R. Mitochondrial bioenergetics and structural network organization. J Cell Sci 2007; 120:838848 .

13. Detmer SA, Chan DC. Functions and dysfunctions of mitochondrial dynamics. Nat Rev Mol Cell Biol 2007; 8:870-879.

14. Hoppeler H, Fluck M. Plasticity of skeletal muscle mitochondria: structure and function. Med Sci Sports Exerc 2003; 35:95-104.

15. Yin F, Cadenas E. Mitochondria: The Cellular Hub of the Dynamic Coordinated Network. Antioxid Redox Signal 2015; 22:961-964.

16. Balaban RS, Nemoto S, Finkel T. Mitochondria, Oxidants, and Aging. Cell 2005; 120:483-495.

17. Chabi B, Ljubicic V, Menzies KJ, Huang JH, Saleem A, Hood DA. Mitochondrial function and apoptotic susceptibility in aging skeletal muscle. Aging Cell 2008; $7: 2-12$

18. Dirks AJ, Hofer T, Marzetti E, Pahor M, Leeuwenburgh C. Mitochondrial DNA mutations, energy metabolism and apoptosis in aging muscle. Ageing Res Rev 2006; 5:179195.

19. Lin MT, Beal MF. Mitochondrial dysfunction and oxidative stress in neurodegenerative diseases. Nature 2006; 443:787795.

20. Peterson CM, Johannsen DL, Ravussin E. Skeletal Muscle Mitochondria and Aging: A Review. J Aging Res 2012; 2012:1-20.

21. Sanz A, Pamplona R, Barja G. Is the Mitochondrial Free Radical Theory of Aging Intact? Antioxid Redox Signal 2006; 8:582-599.

22. Cadenas E, Davies KJA. Mitochondrial free radical generation, oxidative stress, and aging1. Free Radic Biol Med 2000; 29:222-230.

23. Nair KS. Aging muscle. Am J Clin Nutr 2005; 81:953-963.

24. Marzetti E, Leeuwenburgh C. Skeletal muscle apoptosis, sarcopenia and frailty at old age. Exp Gerontol 2006; 41:1234-1238.

25. Johnson ML, Robinson MM, Nair KS. Skeletal muscle aging and the mitochondrion. Trends Endocrinol Metab 2013; 24:247-256.

26. Conley KE, Jubrias SA, Esselman PC. Oxidative capacity and ageing in human muscle. J Physiol 2000; 526:203-210.
27. Joseph A-M, Adhihetty PJ, Wawrzyniak NR, Wohlgemuth SE, Picca A, Kujoth GC, Prolla TA, Leeuwenburgh C. Dysregulation of Mitochondrial Quality Control Processes Contribute to Sarcopenia in a Mouse Model of Premature Aging. PLoS ONE 2013; 8:e69327.

28. Morris G, Maes M. Mitochondrial dysfunctions in Myalgic Encephalomyelitis / chronic fatigue syndrome explained by activated immuno-inflammatory, oxidative and nitrosative stress pathways. Metab Brain Dis 2014; 29:19-36.

29. Wang C-H, Wu S-B, Wu Y-T, Wei Y-H. Oxidative stress response elicited by mitochondrial dysfunction: Implication in the pathophysiology of aging. Exp Biol Med 2013; 238:450-460.

30. Trifunovic A, Larsson N-G. Mitochondrial dysfunction as a cause of ageing: 4th Key Symposium: Mitochondrial dysfunction as a cause of ageing. J Intern Med 2008; 263:167-178.

31. Smits B, van den Heuvel L, Knoop H, Küsters B, Janssen A, Borm G, Bleijenberg G, Rodenburg R, van Engelen B. Mitochondrial enzymes discriminate between mitochondrial disorders and chronic fatigue syndrome. Mitochondrion 2011; 11:735-738.

32. Castro-Marrero J, Cordero MD, Sáez-Francas N, JimenezGutierrez C, Aguilar-Montilla FJ, Aliste L, Alegre-Martin J. Could Mitochondrial Dysfunction Be a Differentiating Marker Between Chronic Fatigue Syndrome and Fibromyalgia? Antioxid Redox Signal 2013; 19:1855-1860.

33. Fulle S, Mecocci P, Fanó G, Vecchiet I, Vecchini A, Racciotti D, Cherubini A, Pizzigallo E, Vecchiet L, Senin $\mathrm{U}$, others. Specific oxidative alterations in vastus lateralis muscle of patients with the diagnosis of chronic fatigue syndrome. Free Radic Biol Med 2000; 29:1252-1259.

34. Kennedy G, Spence VA, McLaren M, Hill A, Underwood $\mathrm{C}$, Belch JJF. Oxidative stress levels are raised in chronic fatigue syndrome and are associated with clinical symptoms. Free Radic Biol Med 2005; 39:584-589.

35. Lerdal A, Wahl AK, Rustoen T, Hanestad BR, Moum T. Fatigue in the general population: A translation and test of the psychometric properties of the Norwegian version of the fatigue severity scale. Scand J Public Health 2005; 33:123130.

36. Adhihetty PJ, Uguccioni G, Leick L, Hidalgo J, Pilegaard $\mathrm{H}$, Hood DA. The role of PGC-1 on mitochondrial function and apoptotic susceptibility in muscle. AJP Cell Physiol 2009; 297:C217-C225.

37. Joseph A-M, Adhihetty PJ, Buford TW, Wohlgemuth SE, Lees HA, Nguyen LM-D, Aranda JM, Sandesara BD, Pahor M, Manini TM, Marzetti E, Leeuwenburgh C. The impact of aging on mitochondrial function and biogenesis pathways in skeletal muscle of sedentary high- and lowfunctioning elderly individuals: Mitochondrial function in elderly individuals. Aging Cell 2012; 11:801-809.

38. Kujoth GC, Hiona A, Pugh TD, Someya S, Panzer K, Wohlgemuth SE, Hofer T, Seo AY, Sullivan R, Jobling 
WA, Morrow JD, Remmen HV, et al. Mitochondrial DNA Mutations, Oxidative Stress, and Apoptosis in Mammalian Aging. Science 2005; 309:481-484.

39. Meissner C. Mutations of mitochondrial DNA - cause or consequence of the ageing process? Z Für Gerontol Geriatr 2007; 40:325-333.

40. Seo AY, Joseph A-M, Dutta D, Hwang JCY, Aris JP, Leeuwenburgh C. New insights into the role of mitochondria in aging: mitochondrial dynamics and more. J Cell Sci 2010; 123:2533-2542.

41. Short KR, Bigelow ML, Kahl J, Singh R, Coenen-Schimke J, Raghavakaimal S, Nair KS. Decline in skeletal muscle mitochondrial function with aging in humans. Proc Natl Acad Sci U S A 2005; 102:5618-5623.

42. Lenaz G, D’Aurelio M, Merlo Pich M, Genova ML, Ventura B, Bovina C, Formiggini G, Parenti Castelli G. Mitochondrial bioenergetics in aging. Biochim Biophys Acta BBA - Bioenerg 2000; 1459:397-404.

43. Donmez G, Guarente L. Aging and disease: connections to sirtuins. Aging Cell 2010; 9:285-290.

44. Broskey NT, Greggio C, Boss A, Boutant M, Dwyer A, Schlueter L, Hans D, Gremion G, Kreis R, Boesch C, Canto C, Amati F. Skeletal Muscle Mitochondria in the Elderly: Effects of Physical Fitness and Exercise Training. J Clin Endocrinol Metab 2014; 99:1852-1861.

45. Kang C, Chung E, Diffee G, Ji LL. Exercise training attenuates aging-associated mitochondrial dysfunction in rat skeletal muscle: Role of PGC-1 $\alpha$. Exp Gerontol 2013; 48:1343-1350.

46. Ljubicic V, Joseph A-M, Adhihetty PJ, Huang JH, Saleem A, Uguccioni G, Hood DA. Molecular basis for an attenuated mitochondrial adaptive plasticity in aged skeletal muscle. Aging (Albany NY). 2009; 1:818. doi: 10.18632/ aging. 100083.

47. McArdle A, Vasilaki A, Jackson M. Exercise and skeletal muscle ageing: cellular and molecular mechanisms. Ageing Res Rev 2002; 1:79-93.

48. Holloway GP, Thrush AB, Heigenhauser GJF, Tandon NN, Dyck DJ, Bonen A, Spriet LL. Skeletal muscle mitochondrial FAT/CD36 content and palmitate oxidation are not decreased in obese women. Am J Physiol Endocrinol Metab 2007; 292:E1782-E1789.

49. Brown PJ, Roose SP, Zhang J, Wall M, Rutherford BR, Ayonayon HN, Butters MA, Harris T, Newman AB, Satterfield S, Simonsick EM, Yaffe K. Inflammation, Depression, and Slow Gait: A High Mortality Phenotype in Later Life. J Gerontol A Biol Sci Med Sci 2016; 71:221227.

50. Zalli A, Jovanova O, Hoogendijk WJG, Tiemeier H, Carvalho LA. Low-grade inflammation predicts persistence of depressive symptoms. Psychopharmacology (Berl) 2016; 233:1669-1678.

51. Finck BN, Kelly DP. PGC-1 coactivators: inducible regulators of energy metabolism in health and disease. $\mathrm{J}$
Clin Invest 2006; 116:615-622.

52. Menzies KJ, Hood DA. The role of SirT1 in muscle mitochondrial turnover. Mitochondrion 2012; 12:5-13.

53. Viña J, Gomez-Cabrera MC, Borras C, Froio T, SanchisGomar F, Martinez-Bello VE, Pallardo FV. Mitochondrial biogenesis in exercise and in ageing. Adv Drug Deliv Rev 2009; 61:1369-1374.

54. Garnier A, Fortin D, Deloménie C, Momken I, Veksler V, Ventura-Clapier R. Depressed mitochondrial transcription factors and oxidative capacity in rat failing cardiac and skeletal muscles. J Physiol 2003; 551:491-501.

55. Wenz T, Rossi SG, Rotundo RL, Spiegelman BM, Moraes $\mathrm{CT}$. Increased muscle PGC- $1 \alpha$ expression protects from sarcopenia and metabolic disease during aging. Proc Natl Acad Sci 2009; 106:20405-20410.

56. Kong X, Wang R, Xue Y, Liu X, Zhang H, Chen Y, Fang F, Chang Y. Sirtuin 3, a New Target of PGC-1 $\alpha$, Plays an Important Role in the Suppression of ROS and Mitochondrial Biogenesis. PLoS ONE 2010; 5:e11707.

57. Agudelo LZ, Femenía T, Orhan F, Porsmyr-Palmertz M, Goiny M, Martinez-Redondo V, Correia JC, Izadi M, Bhat M, Schuppe-Koistinen I, Pettersson AT, Ferreira DMS, et al. Skeletal Muscle PGC-1 $\alpha 1$ Modulates Kynurenine Metabolism and Mediates Resilience to Stress-Induced Depression. Cell 2014; 159:33-45.

58. Kato K, Sullivan PF, Eveng \a ard B, Pedersen NL. Premorbid predictors of chronic fatigue. Arch Gen Psychiatry 2006; 63:1267-1272.

59. Fukuda R, Zhang H, Kim J, Shimoda L, Dang CV, Semenza GL. HIF-1 Regulates Cytochrome Oxidase Subunits to Optimize Efficiency of Respiration in Hypoxic Cells. Cell 2007; 129:111-122.

60. Matoba S, Kang J-G, Patino WD, Wragg A, Boehm M, Gavrilova O, Hurley PJ, Bunz F, Hwang PM. p53 Regulates Mitochondrial Respiration. Science 2006; 312:1650-1653.

61. Srinivasan S, Avadhani NG. Cytochrome c oxidase dysfunction in oxidative stress. Free Radic Biol Med 2012; 53:1252-1263.

62. Chan DC. Fusion and Fission: Interlinked Processes Critical for Mitochondrial Health. Annu Rev Genet 2012; 46:265287.

63. Paradies G, Petrosillo G, Paradies V, Ruggiero FM. Oxidative stress, mitochondrial bioenergetics, and cardiolipin in aging. Free Radic Biol Med 2010; 48:12861295.

64. Westermann B. Mitochondrial fusion and fission in cell life and death. Nat Rev Mol Cell Biol 2010; 11:872-884.

65. Hickie I, Hooker A, Hadzi-Pavlovic D, Bennett B, Wilson A, Lloyd A. Fatigue in selected primary care settings: sociodemographic and psychiatric correlates. Med J Aust 1996; 164:585-588.

66. Broderick JE, Schwartz JE, Vikingstad G, Pribbernow M, Grossman S, Stone AA. The accuracy of pain and fatigue 
items across different reporting periods. PAIN 2008; 139:146-157.

67. Yellen SB, Cella DF, Webster K, Blendowski C, Kaplan E. Measuring fatigue and other anemia-related symptoms with the Functional Assessment of Cancer Therapy (FACT) measurement system. J Pain Symptom Manage 1997; 13:63-74.

68. Chandran V, Bhella S, Schentag C, Gladman DD. Functional Assessment of Chronic Illness Therapy-Fatigue Scale is valid in patients with psoriatic arthritis. Ann Rheum Dis 2007; 66:936-939.

69. Cella D, Lai J, Chang C-H, Peterman A, Slavin M. Fatigue in cancer patients compared with fatigue in the general United States population. Cancer 2002; 94:528-538.

70. Cella D, Yount S, Sorensen M, Chartash E, Sengupta N, Grober J. Validation of the Functional Assessment of Chronic Illness Therapy Fatigue Scale relative to other instrumentation in patients with rheumatoid arthritis. J Rheumatol 2005; 32:811-819.
71. Webster K, Cella D, Yost K. The Functional Assessment of Chronic Illness Therapy (FACIT) Measurement System: properties, applications, and interpretation. Health Qual Life Outcomes 2003; 1:79.

72. Questionnaires. http://www.facit.org/FACITOrg/ Questionnaires. Accessed 7 December 2015.

73. Bergström J. Percutaneous Needle Biopsy of Skeletal Muscle in Physiological and Clinical Research. Scand J Clin Lab Invest 1975; 35:609-616.

74. Radloff LS. The CES-D Scale A Self-Report Depression Scale for Research in the General Population. Appl Psychol Meas 1977; 1:385-401.

75. Folstein MF, Folstein SE, McHugh PR. 'Mini-mental state': A practical method for grading the cognitive state of patients for the clinician. J Psychiatr Res 1975; 12:189-198. 